\title{
Für Freiheit und Handlungsspielraum
}

Ignazio Cassis

Dr. med., MPH, Nationalrat

Der Verband Zürcher Krankenhäuser feiert heute sein 75-jähriges Bestehen.

Es ist mir eine grosse Ehre, als Nicht-Zürcher-Vertreter der Bundespolitik - ja sogar als Nicht-Deutschschweizer - zu diesem Anlass einige Worte an Sie richten zu dürfen. Der Kanton Zürich beweist damit einmal mehr seine Offenheit, über die sprachlichen und kulturellen Grenzen unserer Landesregionen zu blicken. Haben Sie dafür herzlichen Dank!

Obwohl ich früher im Verwaltungsrat des Verbunds der Tessiner Öffentlichen Spitäler - EOC - mitwirken durfte und zuvor als Kantonsarzt zehn Jahre lang eng mit den Spitälern in Kontakt war, liegt mein Fokus heute, als Präsident von CURAVIVA Schweiz, vermehrt auf der Welt der Pflegeheime und anderen sozialen Institutionen. Und, als Präsident des Verbands der innovativen Krankenversicherer curafutura, auf der nachhaltigen Weiterentwicklung unseres solidarisch gestalteten, wettbewerblich orientierten Gesundheitswesens.

Diese Pluralität der Perspektiven ist mir als nationaler Gesundheitspolitiker wichtig. Sie hilft mir, im Dschungel unseres Gesundheitssystems nicht den Überblick zu verlieren und gleichzeitig das eigentliche Ziel nicht aus den Augen zu verlieren: Allen Einwohnerinnen und Einwohnern unseres Landes die bestmögliche medizinische Behandlung zu ermöglichen, mit einem solidarischen, nachhaltig finanzierbaren System. Im Zentrum dieses Systems steht dabei immer der Patient, der für mich als Liberaler ein mündiger Bürger ist. Mündig heisst, dass er zuerst für seine Gesundheit und Krankheit verantwortlich ist. Erst wenn im Krankheitsfall die Kosten der richtigen Behandlungen seine finanziellen Möglichkeiten übersteigen, ist die obligatorische Mitfinanzierung seiner Mitmenschen gefragt - die rechtlich organisierte Solidarität.

Ich bin mir bewusst, dass diese Auslegung der gesellschaftlichen Solidarität längst nicht mehr Allgemeingut ist. Wobei alle Gesundheitssysteme Europas hier eine ähnliche Entwicklung aufzeigen: Sie basieren auf einem grundsätzlichen - und besorgniserregenden Missverständnis, nämlich auf dem Ungleichgewicht zwischen Rechten und Pflichten, zwischen Freiheit und Eigenverantwortung. Die Schweiz macht hier keine Ausnahme. Mit dem Unterschied, dass diese Entwicklungen, mit all ihren negativen Folgen, von der direkten Demokratie zum Glück etwas gebremst wer-

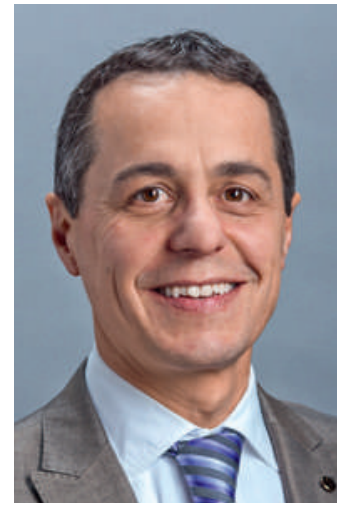

Ignazio Cassis: «Es gibt ein Ungleichgewicht zwischen Rechten und Pflichten, zwischen Freiheit und Eigenverantwortung.»

den. Kein Wunder also, dass die Finanzierung unseres Gesundheitssystems, mit den Spitälern als dessen kostspieligstes Element, ein Dauerbrenner ist.

Die Situation widerspiegelt die Entwicklung in Europa nach dem Zweiten Weltkrieg: Die Illusion, dass alle noch so persönlichen medizinischen Wünsche - die in einer reichen Gesellschaft per definitionem einem Bedürfnis entsprechen - solidarisch zu finanzieren sind. Wie schmerzhaft heute der Versuch der Politik ist, diese entgleiste Entwicklung zu korrigieren, brauche ich Ihnen nicht zu erzählen.

Noch ist von einer Strukturbereinigung unserer übersättigten Spitallandschaft noch relativ wenig zu spüren.

Tatsächlich ging auch unsere wichtigste Reform der letzten 20 Jahre - die Einführung des Krankenversicherungsgesetzes KVG - in die falsche Richtung, mindestens was die Kostenkontrolle anbelangt. Und diese war - nota bene - der wichtigste Grund jener Reform. Umso bedeutender dann die Korrektur vom Dezember 2007, die Einführung der neuen Spitalfinanzierung, die nun nach vier Jahren langsam zum Tragen kommt. Fast ein Wunder, dass dieser zentrale Eingriff in das System damals ohne Referendum gelungen ist! Die dual-fixe Finanzierung hat die Wettbewerbsverzerrung zwischen öffentlichen und privaten Trägerschaften abgeschafft, die Abrechnung mit Fallpauschalen die Vergleichbarkeit und Transparenz der Kosten verbessert. Und all dies - das wissen wir heute - ohne blutige Entlassungen oder regionale Versorgungsgefährdung.

Doch noch ist von einer Strukturbereinigung unserer übersättigten Spitallandschaft noch relativ wenig zu spüren. Solche Reformen brauchen mindestens zehn 
Jahre, bis es möglich ist, Bilanz zu ziehen. Ich bin der Meinung, wir sind heute auf gutem Weg und Ihr Verband kann mit Stolz zurückblicken: Sie haben Pionierarbeit geleistet und sind heute besser aufgestellt als manch andere Kantone. Das ist für Zürich kein Novum und sorgt anderswo für gewisse Irritation - der Preis für die undankbare Vorreiterrolle!

Wohin führt die Reise jetzt? In welche Zukunft blicken die 31 Mitglieder des Zürcher Spitalverbands mit ihren mehr als 30000 Mitarbeiterinnen und Mitarbeitern? Ich bin kein Kaffeesatzleser, trotzdem wage ich eine Prognose. Ich denke, drei Trends werden die nächsten zehn Jahre dominieren:

\section{Ein Blick in die Zukunft}

\section{Spitäler werden eigenständige Unternehmen, die zueinander im Kosten- und Qualitätswett- bewerb stehen.}

Vom Mittelalter bis zum 19. Jahrhundert waren die Spitäler vor allem ein Schutzraum für schwache, bedürftige und obdachlose Menschen, die von der privaten Solidarität - oft religiöse Orden - getragen wurden. Im 20. Jahrhundert sind sie zum grossen Teil vom Staat (Kantone und Gemeinde) übernommen worden und haben sich zur Hightech-Drehscheibe unserer Gesundheitssysteme entwickelt. Meist auf einem Hügel gebaut, so wie in früheren Zeiten die Kathedralen, dominierten sie die Stadt. Ihre symbolische Bedeutung war somit stark, sie hatten quasi religiösen Charakter. Kein Wunder, ist die Schliessung eines Spitals immer auch ein gesellschaftliches Trauma. Im 21. Jahrhundert werden die Spitäler Bestandteile grosser integrierter Versorgungsnetze, die zunehmend mit komplexen Pauschalen vergütet werden, welche die Vertragspartner miteinander aushandeln. Damit die Vertragspartner dieser Aufgabe gewachsen sind, muss ihre Zusammenarbeit künftig aber konstruktiver werden, als dies heute der Fall ist. Die Kantone werden sich vermehrt auf ihre regulatorische Rolle und auf ihre Aufsichtsfunktion zurückziehen, diese hingegen stärker wahrnehmen.

\section{Die organisatorische und finanzielle Trennung ambulant vs. stationär wird verschwinden.}

In den 70er Jahren reichten 2,5 Personen an klinischem Personal, um einen durchschnittlichen stationären $\mathrm{Pa}$ tienten behandeln zu können. 20 Jahre später waren es bereits 15 Beteiligte. Heute kommt man leicht auf 50 und mehr: vom einweisenden Arzt bis zur Spitalärztin, von der Spitex-Fachperson über Apotheker, Radiologe, Operateur, Konsiliarius bis hin zu Pflegefachpersonen oder Physiotherapeuten. Die Anzahl Gesundheitsfach- leute pro Patient - ambulant und stationär - hat also exponentiell zugenommen, die Behandlung ist entsprechend komplexer und fragmentierter geworden. Die Trennung ambulant/stationär ist nicht mehr klar, und das Spital bietet vermehrt beide Optionen an; die Übergänge sind fliessend. Ob eine Leistung stationär oder ambulant erbracht wird, hat zudem schon heute oft mehr mit finanziellen Anreizen als mit der ärztlichen Beurteilung zu tun. Solche Anreize müssen korrigiert werden: Eine gleiche Finanzierung ambulant/ stationär wird uns hier weiterführen. Das Stichwort hierzu heisst Monismus.

\section{Der Wettbewerb wird internationaler, nationale Allianzen entstehen.}

Die Mobilität in der Bevölkerung nimmt kontinuierlich $\mathrm{zu}$, demzufolge auch diejenige der Patientinnen und Patienten. Neue Instrumente im Bereich eHealth - und entsprechende Rahmenbedingungen wie das Bundesgesetz über das elektronische Patientendossier - ermöglichen die Standardisierung und weltweite Vermittlung von Patienteninformationen.

Neue gesetzliche Bestimmungen werden das Territorialitätsprinzip lockern - eine entsprechende KVG-Änderung war bis Mitte Februar 2015 in der Vernehmlassung. Bereits seit 2006 werden Pilotprojekte zur Kostenübernahme von Leistungen im grenznahen Ausland durchgeführt. Beide Elemente werden die medizinische Behandlung auch im Ausland erleichtern. Nicht nur die Spitzenmedizin wird also zunehmend im internationalen Wettbewerb stehen, sondern auch die - oft planbare - Versorgung der chronisch Kranken. Spitäler werden sich hier alliieren müssen - sogar verschmelzen - um unter stärkerem Wettbewerbsdruck erfolgreich zu sein.

\section{Fazit und Bitte}

Die Zukunft im Gesundheitswesen bleibt in jedem Fall spannend. Ethische und organisatorische Fragen werden die nächsten Jahre prägen. Die Frage nach der ethischen Verantwortung wird zentral, genauso wie die Suche nach neuen integrierten Versorgungsformen, die den historisch gewachsenen Dualismus ambulant/ stationär zunehmend abschaffen werden. Der Verband der Zürcher Krankenhäuser wird auch in Zukunft gefordert sein, unser bewährtes Gesundheitssystem aktiv und nachhaltig zu prägen und weiterzuentwickeln Meine Bitte: Nehmen Sie diese Herausforderung an! Delegieren Sie die Aufgabe nicht an Beamte und Politiker. Nur so können Sie auch in den nächsten 75 Jahren sicherstellen, dass Ihre Freiheit und Ihr Handlungsspielraum garantiert sind.

Das wünsche ich Ihnen, das wünsche ich uns allen! 